\title{
Structure Watch
}

\section{UNDERSTANDING $\alpha$-CATENIN INTERACTIONS}

$\alpha$-catenin monomers interact with $\beta$-catenin-cadherin complexes to stabilize adherens junctions between cells. As $\alpha$-catenin homodimers interact with $\mathrm{F}$-actin, $\alpha$-catenin must link $\beta$-catenin-cadherin to $\mathrm{F}$-actin through its interaction with other proteins, such as the F-actin-binding protein vinculin. Here, Rangarajan and Izard report the crystal structure of nearly full-length human $\alpha$-catenin at $3.7 \AA$ resolution, revealing how $\alpha$-catenin makes these specific interactions. They show that $\alpha$-catenin forms an asymmetric dimer, with each subunit comprised of four helix-bundle domains that participate in specific intermolecular interactions. This results in a left handshake-like dimer in which the two monomers have distinct conformations. Analysis shows that the F-actin-binding surface of $\alpha$-catenin involves intermolecular interactions between each subunit of the $\alpha$-catenin dimer; these interactions are disrupted by $\alpha$-catenin- $\beta$-catenin interactions. This explains why monomeric $\alpha$-catenin cannot bind $\mathrm{F}$-actin and why $\beta$-catenin interacts with $\alpha$-catenin monomers. The asymmetry of $\alpha$-catenin also regulates its interaction with activated vinculin; the dimer first interacts with one vinculin monomer before forming a 2:2 a-catenin:vinculin heterotetrameric complex that can bind F-actin. Thus, the dimer asymmetry of $\alpha$-catenin is important for its ability to bind F-actin and to interact with activated vinculin. ORIGINAL RESEARCH PAPER Rangarajan, E. S. \& Izard, T. Dimer asymmetry defines
a-catenin interactions. Nature Struct. Mol. Biol. 6 Jan 2013 (doi:10.1038/nsmb.2479)

\section{DISSECTING TFIID ASSEMBLY}

Association of the transcription factor TFIID with gene promoters drives the formation of the pre-initiation complex during RNA polymerase II-mediated transcription. TFIID consists of TATA-binding protein (TBP) and 13 TBP-associated factors (TAFs). How does the TFIID complex assemble? Previous studies in Drosophila melanogaster and yeast have led to a model in which a core-TFIID subcomplex forms initially that has twofold symmetry and contains duplicate copies of particular TAFs; addition of TBP and the remaining TAFs is thought to produce an asymmetric holo-TFIID. Here, Bieniossek et al. determine the structure of human core-TFIID complexes using wild-type or mutant TAFs and cryo-electron microscopy. Consistent with previous studies, they show that the core TFIID has twofold symmetry, and the authors were able to assign the conserved domains of the TAFs within this structure. They also provide insights into the transition of this complex to the asymmetric structure that is characteristic of the holo-TFIID by showing that the addition of one TAF8-TAF10 complex is sufficient to induce asymmetry. This supports a model of stepwise holo-TFIID assembly from the core-TFIID complex.

ORIGINAL RESEARCH PAPER Bieniossek, C. et al. The architecture of human genera transcription factor TFIID core complex. Nature 6 Jan 2013 (doi:10.1038/nature11791) 